University of Nebraska - Lincoln

DigitalCommons@University of Nebraska - Lincoln

Faculty Publications, Department of Physics and Astronomy

Research Papers in Physics and Astronomy

2007

\title{
Acoustic Analog to Quantum Mechanical Level Splitting
}

Shawn A. Hilbert

University of Nebraska-Lincoln, shilbert@berry.edu

Herman Batelaan

University of Nebraska - Lincoln, hbatelaan@unl.edu

Follow this and additional works at: https://digitalcommons.unl.edu/physicsfacpub

Part of the Physics Commons

Hilbert, Shawn A. and Batelaan, Herman, "Acoustic Analog to Quantum Mechanical Level Splitting" (2007). Faculty Publications, Department of Physics and Astronomy. 103.

https://digitalcommons.unl.edu/physicsfacpub/103

This Article is brought to you for free and open access by the Research Papers in Physics and Astronomy at DigitalCommons@University of Nebraska - Lincoln. It has been accepted for inclusion in Faculty Publications, Department of Physics and Astronomy by an authorized administrator of DigitalCommons@University of Nebraska Lincoln. 


\title{
Acoustic analog to quantum mechanical level splitting
}

\author{
Shawn A. Hilbert ${ }^{\mathrm{a})}$ and Herman Batelaan \\ Department of Physics and Astronomy, University of Nebraska-Lincoln, 116 Brace Laboratory, Lincoln, \\ Nebraska 68588-0111
}

(Received 26 May 2006; accepted 14 July 2007)

\begin{abstract}
A simple physical system is discussed that mirrors the quantum mechanical infinite square well with a central delta well potential. The physical realization consists of a continuous sound wave traveling in a pair of tubes separated by an adjustable diaphragm. The equivalence between the quantum system and the acoustic system is explored. The analytic solution to the quantum system exhibits level splitting as does the acoustic system. (C) 2007 American Association of Physics Teachers.
\end{abstract}

[DOI: $10.1119 / 1.2772278$ ]

\section{INTRODUCTION}

A common system of interest in quantum mechanics is the infinite square well. A typical exercise in introductory quantum mechanics courses is finding the energy eigenvalues and eigenfunctions for such a system. ${ }^{1-3}$ In more advanced courses, the infinite square well is used as a starting point for perturbation theory. ${ }^{4,5}$ One such perturbation is the introduction of a delta function potential at the midpoint of the well. ${ }^{4}$

In this paper we discuss an acoustic analog of an infinite square well with an adjustable central delta function potential well. The acoustic system is comprised of two connected tubes of length $a$ (Fig. 1). This system forms one long, closed tube of length $L=2 a$ supporting integer numbered resonances. Quantum mechanically, this system can be thought of as an infinite potential well supporting a discrete set of stationary states. In the center of the acoustic system, we place a thin aluminum disk with an adjustable hole. Quantum mechanically, this disk can be represented by a delta function potential. As the hole in the disk becomes smaller (the strength of the delta function potential increases,) the odd order resonant frequencies (energy eigenvalues) shift, while the even order resonant frequencies remain the same. When the connecting disk becomes solid (the delta function potential has infinite strength), the odd order resonant frequencies merge with the even orders. These eigenvalues coincide with that of a tube of length $a$, half that of the original tube. The system in this limiting case consists of two uncoupled tubes (infinite potential wells) of half the length. (This phenomenon is level merging. Starting with the degenerate levels and increasing the hole size gives level splitting.)

This experiment can be used to compare acoustic resonances and quantum mechanical energy eigenvalues and to explore perturbation theory and level splitting. The simplicity of the experiment makes this system suitable for laboratory courses and lecture demonstrations.

\section{THEORY}

\section{A. Formal equivalence}

It is useful to first explore the general connection between wave propagation in quantum mechanics and sound. The equation for the propagation of sound in the acoustic limit (small displacement amplitude) in liquids and gases is given by ${ }^{6,7}$

$$
\nabla^{2} \xi=\frac{1}{v_{s}^{2}} \frac{\partial^{2}}{\partial t^{2}} \xi
$$

where $\xi$ is the displacement, and the speed of sound $v_{s}$ is dependent on the impedance and density of the medium. If we assume sinusoidal temporal behavior for the displacement, the time and spatial variables can be separated. This assumption and the acoustic dispersion relation $k=\omega / v$ results in the time-independent differential equation

$$
\nabla^{2} \xi+k^{2} \xi=0 .
$$

The time-independent Schrödinger equation may be written as

$$
\nabla^{2} \varphi+\frac{2 m}{\hbar^{2}}(E-V) \varphi=0
$$

where a time-independent potential and scalar wave function are assumed. If we substitute $k=\sqrt{2 m(E-V) / \hbar^{2}}$ in Eq. (3), we obtain an equation that is formally identical to Eq. (2).

Equation (3) is also identical to the Helmholtz equation, which yields analogies between sound, quantum mechanics, and optics. An optical element can be specified by its spatially varying index of refraction. In quantum mechanics, different physical systems can be modeled by an appropriate choice of the spatially varying potential. For sound waves, physical systems can be defined by spatially varying impedances. ${ }^{8}$ For example, just as a shaped piece of glass can be a lens for light, a focused laser can be a lens for matter waves, ${ }^{9}$ and a carbon dioxide-filled balloon acts like a lens for sound waves. ${ }^{10}$

\section{B. Quantum eigenenergies and sound resonances}

We compare the acoustic resonances in a closed tube and the quantum mechanical eigenfrequencies of an infinite square well. The corresponding differential equation is

$$
\frac{d^{2}}{d x^{2}} \varphi+\frac{2 m E}{\hbar^{2}} \varphi=0 .
$$

For a potential well of length $L=2 a$, the solutions are $k_{n}$ $=n \pi / L$, where $n$ is an integer. The relation of $k$ to the energy gives the energy eigenvalues

$$
E_{n}=\frac{n^{2} \pi^{2} \hbar^{2}}{2 m L^{2}}=\frac{n^{2} \pi^{2} \hbar^{2}}{8 m a^{2}}, \quad \text { where } n=1,2,3, \ldots
$$

The relation of $k$ to the frequency gives the resonance frequencies, 


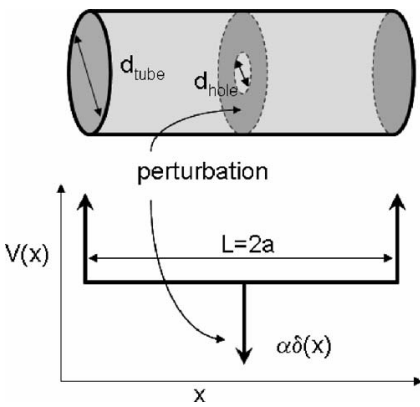

Fig. 1. The displacement wave and resonances are investigated for an acoustic system consisting of two tubes separated by an adjustable diaphragm The displacement wave and resonances are analogous to the wave functions and eigenenergies of an infinite potential well with an adjustable delta function potential well perturbation.

$$
f_{n}=n v /(2 L)=n v /(4 a) .
$$

The normalized eigenfunctions are given by

$$
\varphi_{n}(x)=\sqrt{\frac{2}{L}} \sin \left(\frac{n \pi x}{L}\right)=\sqrt{\frac{1}{a}} \sin \left(\frac{n \pi x}{2 a}\right) .
$$

The wave functions, $\varphi_{n}$, for the odd (even) order states are symmetric (antisymmetric) with respect to the center of the well $(x=a)$.

The acoustic resonances of a closed tube also follow Eq. (6). The acoustic displacement standing wave is

$$
\xi(x)=\xi_{\max } \sin \left(\frac{n \pi x}{2 a}\right),
$$

for the $n$th resonance. Equation (8) is the same shape as the quantum mechanical wave function. The pressure wave (which is actually measured by a microphone) is proportional to the derivative of the displacement wave and has the form

$$
p(x)=p_{\max } \cos \left(\frac{n \pi x}{2 a}\right) .
$$

\section{Reflection from a finite barrier}

The perturbation for the infinite potential well is a variable strength delta function potential well. For the acoustic system the perturbation is a disk with a variable diameter hole. To establish the analogy in more detail, a connection can be made between the descriptions of a disk with a finite width hole and the infinitesimally thin delta function potential. For a sound wave the reflection from a disk with a thin hole can be found by considering three adjacent regions of different impedances. Quantum mechanically, wave reflection from a delta function potential is a textbook problem. ${ }^{4}$

For a sound wave, the reflection coefficient of a boundary between two regions is given by

$$
r_{i j}=\frac{Z_{i}-Z_{j}}{Z_{i}+Z_{j}}
$$

where $Z_{i}$ is the impedance of region $i .^{11}$ If a second boundary follows this first boundary, as is the case for a thin disk, then the reflection coefficient changes. Assuming the outer regions are the same, the reflection caused by the combination of boundaries is ${ }^{11}$

$$
r_{121}=\frac{r_{12}\left(1-e^{2 i k b}\right)}{1-r_{12}^{2} e^{2 i k b}},
$$

where $r_{12}$ is the reflection coefficient of the first boundary alone, $k$ is the wave vector of the sound wave in the central region, and $b$ is the width of the central region. [Note that Eqs. (10) and (11) are similar to the results for the Fresnel equations, where impedances are substituted by indexes of refraction. $^{12}$ ]

The acoustic system has three regions as well: The two outer regions have a cross sectional diameter equal to the tube diameter, and the region inside the disk has a cross sectional diameter of the size of the hole. The impedance inside a tube is given by

$$
Z_{i}=\rho v_{s} / \pi\left(\frac{d_{i}}{2}\right)^{2},
$$

where $d_{i}$ is the open diameter of region $i$, and $\rho$ is the equilibrium density of the medium. If we assume that the medium in which the sound travels is the same throughout the tube, Eq. (10) may be rewritten as

$$
r_{12} \equiv \frac{d_{\text {hole }}^{2}-d_{\text {tube }}^{2}}{d_{\text {hole }}^{2}+d_{\text {tube }}^{2}}
$$

For the quantum case, a particle is incident on a delta well $V=-\alpha \delta(x)(\alpha>0)$ located at $x=0$. The delta function potential well can be approached by a square potential well of depth $V_{0}$ and width $b=1 / V_{0}$, in the limit $b \rightarrow 0$. The continuity of the wave function and its derivative at the boundaries leads to the result

$$
r=\frac{\frac{k_{1}-k_{2}}{k_{1}+k_{2}}\left(1-e^{2 i k_{2} b}\right)}{1-\left(\frac{k_{1}-k_{2}}{k_{1}+k_{2}}\right)^{2} e^{2 i k_{2} b}},
$$

where $k_{1}$ is the wave vector in the regions where $V=0$, and $k_{2}$ is the wave vector in the region within the well. The reflection of a potential step (the first boundary of the potential) is given by ${ }^{13}$

$$
r_{i j}=\frac{k_{i}-k_{j}}{k_{i}+k_{j}} .
$$

The substitution of Eq. (15) into Eq. (14) gives the total reflection of the well

$$
r=\frac{r_{12}\left(1-e^{2 i k_{2} b}\right)}{1-r_{12}^{2} e^{2 i k_{2} b}}
$$

which matches Eq. (11), the reflection for sound.

For the quantum case, Eq. (16) can be simplified by taking the limit $b$ goes to zero. This limit yields the exact reflection coefficient of a delta well (see the appendix):

$$
r=-\frac{m \alpha}{i k \hbar^{2}+m \alpha}
$$

where $k_{2}=\sqrt{2 m(E-V) / \hbar^{2}}=\sqrt{k^{2}+2 m \alpha / b \hbar^{2}}$. Equation gives the quantum reflectivity

$$
R \equiv|r|^{2}=\frac{m^{2} \alpha^{2}}{k^{2} \hbar^{4}+m^{2} \alpha^{2}} .
$$


To complete the connection between the quantum and acoustic systems, an expression for the wave vector inside the disk is needed. A heuristic expression (motivated by the quantum mechanical expression for the wave vector) that gives excellent agreement is

$$
k_{\mathrm{eff}}=\sqrt{k_{s}^{2}+\beta \frac{d_{\mathrm{tube}}^{2}-d_{\mathrm{hole}}^{2}}{d_{\text {tube }}^{2}}},
$$

where $k_{s}$ is the source wave vector, and $\beta$ is a fitting parameter. A formal derivation of Eq. (19) would require the dynamical description of a three-dimensional diaphragm and its effect on the sound wave propagation in one dimension. Such a derivation is beyond the scope of this paper. A single fitting parameter of $\beta=200$ yields good agreement between the quantum eigenfrequencies and the acoustic resonant frequencies for all hole sizes and orders. We replace $k$ by $k_{\text {eff }}$ in Eq. (11) and express the acoustic reflectivity as

$$
R=\left|r_{121}\right|^{2}=\frac{2 r_{12}^{2}\left(1-\cos \left(2 k_{\mathrm{eff}} b\right)\right)}{1-2 r_{12}^{2} \cos \left(2 k_{\mathrm{eff}} b\right)+r_{12}^{2}}
$$

\section{Perturbative and analytic solutions}

The wave functions and eigenenergies can be found perturbatively or analytically. The delta function potential perturbation is

$$
W=-\alpha \delta\left(x-\frac{L}{2}\right)
$$

where $\alpha$ is the strength of the delta well. The perturbation shifts the energy eigenvalues by ${ }^{14}$

$$
\Delta E_{n}=\left\langle\varphi_{n}^{0}|W| \varphi_{n}^{0}\right\rangle
$$

where $\varphi_{n}^{0}$ corresponds to the $n$th unperturbed energy level. Thus, the change in energy for the $n$th energy eigenvalue is

$$
\Delta E_{n}=-\frac{2 \alpha}{L} \int \sin ^{2}\left(\frac{n \pi x}{L}\right) \delta\left(x-\frac{L}{2}\right) d x=-\frac{\alpha}{a} \sin ^{2}\left(\frac{n \pi}{2}\right) .
$$

The perturbation has no effect on the even energy levels because the sine function is zero for even $n$. The odd energy levels decrease by $\alpha / a$.

These solutions can be compared to the exact solutions. The time-independent Schrödinger equation is

$$
-\frac{\hbar^{2}}{2 m} \nabla^{2} \varphi-\alpha \delta\left(x-\frac{L}{2}\right) \varphi=E \varphi, \quad(0<x<L) .
$$

Equation (24) can be solved by matching the boundary conditions for the trial solutions. The infinite potential well is broken into two regions (region I to the left and region II to the right of the delta function). Two boundary conditions come from the requirement that the wave function be zero at the boundaries $(x=0$ and $x=L)$ of the infinite potential well. The continuity of the wave function and the behavior of its derivative at the delta function provide the other boundary conditions. Integration of the Schrödinger equation over a small distance around the delta function leads to the boundary condition

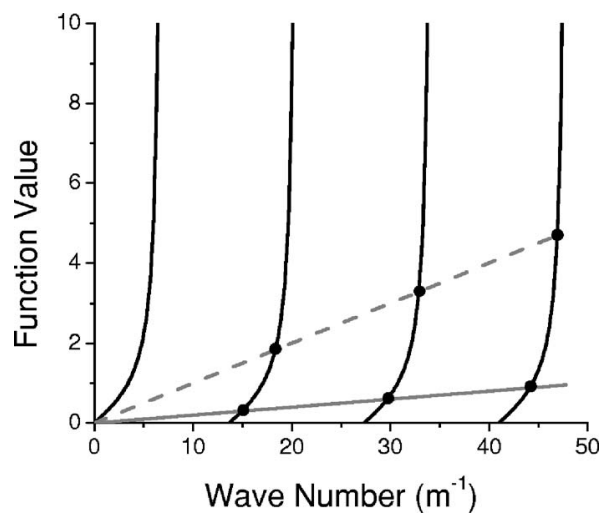

Fig. 2. The analytic solutions for the wave number in an infinite potential well with a central delta function potential are found from Eq. (27). Solutions corresponding to the first three eigenenergies given by $E_{n}$ $=\hbar^{2} k^{2} /(2 m)$ are indicated with dots. Plotted are $\tan (k a / 2)$ (black line) and $k \hbar^{2} /(m \alpha)$ with barrier strengths $\alpha=50 \hbar^{2} / m$ (solid gray) and $\alpha=10 \hbar^{2} / m$ (dashed).

$$
\frac{d}{d x} \varphi\left(\frac{L^{+}}{2}\right)-\frac{d}{d x} \varphi\left(\frac{L^{-}}{2}\right)=-\frac{2 m \alpha}{\hbar^{2}} \varphi\left(\frac{L}{2}\right) .
$$

Thus, the four boundary conditions for this system are $\varphi_{\mathrm{I}}(0)=0, \varphi_{\mathrm{II}}(L)=0, \varphi_{\mathrm{I}}(L / 2)=\varphi_{\mathrm{II}}(L / 2)$, and

$$
\frac{d}{d x} \varphi_{\mathrm{II}}\left(\frac{L}{2}\right)-\frac{d}{d x} \varphi_{\mathrm{I}}\left(\frac{L}{2}\right)=-\frac{2 m \alpha}{\hbar^{2}} \varphi\left(\frac{L}{2}\right) .
$$

Imposing the boundary conditions on the usual exponential trial solutions yields

$$
\frac{k \hbar^{2}}{m \alpha}=\tan (k a) .
$$

Examples of solutions to Eq. (27) are found graphically in Fig. 2 and give the energy eigenvalues $E=\hbar^{2} k^{2} / 2 m$.

There is another set of solutions to consider. If the wave function at the delta function is zero, Eq. (26) becomes

$$
\frac{d}{d x} \varphi_{\mathrm{I}}\left(\frac{L}{2}\right)=\frac{d}{d x} \varphi_{\mathrm{II}}\left(\frac{L}{2}\right) .
$$

If we change the boundary conditions of the system by substituting Eq. (28) for Eq. (26), we obtain the solutions

$$
\sin (k L)=0 \text {. }
$$

This set of solutions is independent of the perturbation. This independence agrees with the perturbative approach; only half the eigenenergies shift, while the other half remain the same with changes in the delta function strength.

An approximate solution to Eq. (27) can be found by using a series expansion. The result is ${ }^{15}$

$$
E \approx \frac{n^{2} \pi^{2} \hbar^{2}}{8 m a^{2}}-\frac{\alpha}{a},
$$

which matches the shift in the energy eigenvalues found from the perturbative approach.

The limit $\alpha$ goes to zero describes an infinite potential well of width $2 a$. This limit for Eqs. (27) and (29) gives the solutions $k_{n}=\left(n+\frac{1}{2}\right) \pi / a$ and $k_{n}=n \pi / a$, respectively. These solutions combine to form $k_{n}=n \pi /(2 a)$, which are the solutions for an infinite potential well of width $2 a$. If the value of 


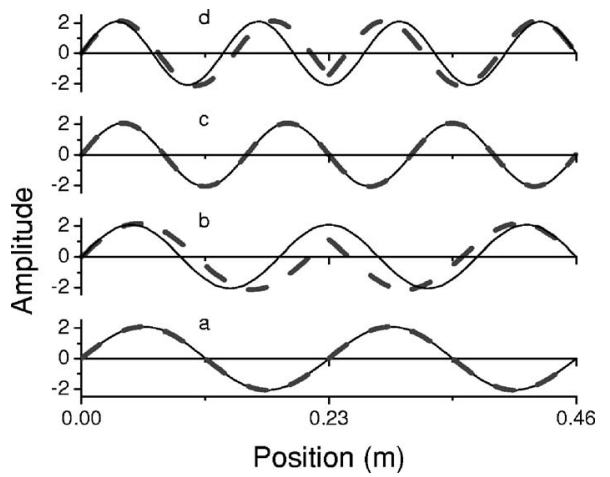

Fig. 3. The normalized wave functions are found for the fourth through seventh (a)-(d) eigenenergies of an infinite potential well of $46 \mathrm{~cm}$ long. The solid lines indicate the wave function of the unperturbed system. The dashed lines are the wave functions when a delta function potential perturbation of strength $\alpha=50 \pi \hbar^{2} /(2 m)$ is introduced. Kinks develop for the odd eigenfunctions (b) and (d) as their eigenenergies shift.

$\alpha$ is taken to go to infinity, both Eqs. (27) and (29) give the solutions $k_{n}=n \pi / a$. These solutions match those for an infinite potential well of width $a$.

The normalized wave functions corresponding to the exact solutions from Eq. (27) are

$$
\begin{aligned}
\varphi_{\mathrm{I}}(x) & =\sqrt{\frac{2 k}{k L-\sin (k L)}} \sin (k x) \\
& =\sqrt{\frac{2 k}{2 k a-\sin (2 k a)}} \sin (k x), \\
\varphi_{\mathrm{II}}(x) & =-\sqrt{\frac{2 k}{k L-\sin (k L)}} \sin [k(x-L)] \\
& =-\sqrt{\frac{2 k}{2 k a-\sin (2 k a)}} \sin [k(x-2 a)] .
\end{aligned}
$$

For the limit $\alpha$ goes to zero, the wave functions are $\varphi_{\mathrm{I}, n}(x)$ $=\sqrt{2 / L} \sin (n \pi x / L) \quad$ and $\quad \varphi_{\mathrm{II}, n}(x)=-\sqrt{2 / L} \sin [n \pi(x / L-1)]$, matching the wave functions for an infinite potential well of width $2 a$. In the limit $\alpha$ goes to infinity, the wave functions become $\varphi_{\mathrm{I}, n}(x)=\sqrt{1 / a} \sin (n \pi x / a)$ and $\varphi_{\mathrm{II}, n}(x)$ $=-\sqrt{1 / a} \sin [n \pi(x / a-1)]$, which is similar to the wave function for an infinite potential well with length $a$. In between the two limiting cases, the wave function develops a kink at the delta function potential well (see Fig. 3).

\section{EXPERIMENT}

\section{A. Apparatus}

The acoustic system is created by linking PVC tube sections with length $a=23 \mathrm{~cm}$ and diameter of 2 in. Both ends of the tubular array are closed with a thin, solid aluminum disk. A microphone (Horn, 252-EM4530-44) and speaker (Kobitone, 253-5151) are mounted within the tube. The measured pressure wave amplitude depends on the positions of the speaker and microphone; resonances cannot be detected when the microphone is placed at a node of a standing wave. A function generator is used to send a sinusoidal wave to the speaker. The microphone picks up the pressure wave in the tube. The microphone signal is amplified (a simple stereo

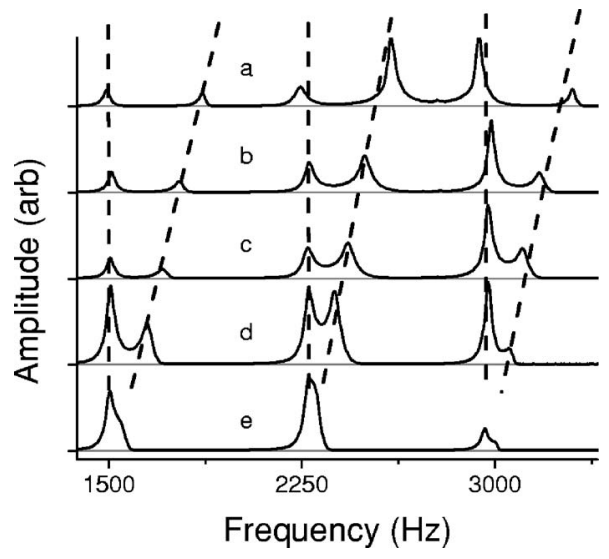

Fig. 4. Measured frequency spectra are shown for two cavities of length $a$ $=23 \mathrm{~cm}$ coupled by a central disk with holes of different diameters: (a) open disk, (b) $24 \mathrm{~mm}$, (c) $17 \mathrm{~mm}$, (d) $13 \mathrm{~mm}$, (e) solid disk. When there is no central disk, the resonances are equally spaced, in accord with the resonances of one cavity of length $2 a$. Introducing a disk with a hole causes the resonances associated with odd eigenfunctions to shift. As the size of the hole becomes smaller, these resonances move to lower frequencies. Once the disk is solid, the shifting resonances merge with the resonances associated with uncoupled cavities of length $a$.

amplifier or an SRS (SR560) high-pass/low-pass voltage amplifier), rectified, and time averaged with an RC filter. The resulting signal is processed by a data acquisition board and recorded. Based on the parameters of the system and assuming a sound velocity of $345 \mathrm{~m} / \mathrm{s}$, the resonance frequencies are expected at integer multiples of $750 \mathrm{~Hz}$.

Our system uses two tube sections. A thin aluminum disk of the same diameter as the tube may be placed between these tube sections. The disk has an adjustable central hole. The size of the hole in the connecting disk controls the coupling between cavities. Using a solid disk effectively decouples the cavities. If no disk is placed between the two tube sections, the system becomes one tube of twice the length. A disk with a hole provides partial coupling between the cavities. We will present data using disks with 8, 13, 17, $21,24,31,37$, and $41 \mathrm{~mm}$ diameter holes.

To find the sound amplitude in the cavity array as a function of wave frequency, a voltage ramp is sent to the voltage controlled frequency input of a function generator. The function generator signal drives a speaker. As the speaker chirps, the wave pressure amplitude is recorded. (Alternatively, a white noise spectrum combined with a Fourier transform can be used.)

The spatial dependence of the sound wave is measured with a microphone attached to a movable, long, thin rod. This rod enters the cavity array through a small hole cut into a disk that closes one end of the tubular array. The position of the microphone can be changed by pushing the rod further into the cavity. This arrangement allows the amplitude to be measured at multiple positions within the tubes, and gives a representation of the standing wave for the acoustic system.

\section{B. Results}

\section{Acoustic spectra}

The frequency spectra are measured for two coupled tubes with lengths of $23 \mathrm{~cm}$ each. The tubes are coupled by a disk with an adjustable hole diameter. In Fig. 4 frequency spectra for different hole diameters are shifted vertically to avoid 


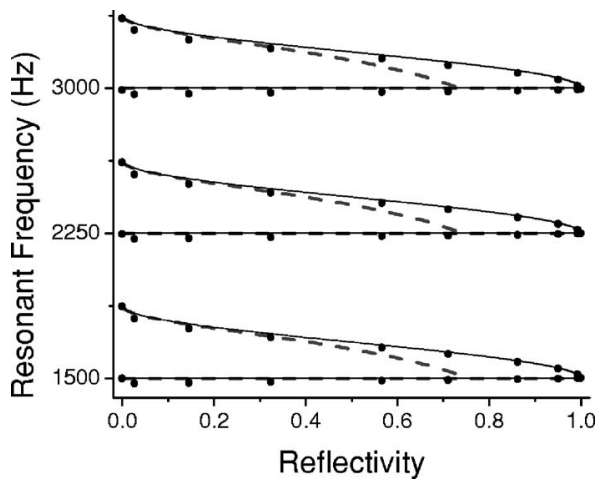

Fig. 5. The measured resonant frequencies (circles) of the acoustic system, and the analytic eigenfrequencies (solid line) are compared as a function of reflectivity. The perturbative eigenenergies (dashed line) are also given. For the resonances, the reflectivity is related to the hole size; for the eigenfrequencies, the reflectivity is related to the delta function potential strength. The three results display level splitting.

overlap. The frequency spectra are displayed over a limited range. At lower frequencies the interpretation of the spectra is complicated by the nonlinearity of the microphone's response curve. At higher frequencies the presence of other resonances, such as transverse modes, complicates the observed spectrum.

In the absence of a central disk, equally spaced resonances are observed. The frequency spacing between two adjacent resonances is about $375 \mathrm{~Hz}(\approx v / 2 L)$. Resonances associated with odd eigenfunctions shift. For decreasing hole size the resonances shift to lower frequencies. The dashed lines in Fig. 4 provide a guide to the eye. For a solid disk the shifting resonances merge at frequencies that match the resonances of an uncoupled tube of length $a$. A double peak is still visible for the solid disk because the reflectivity for this disk is not unity. Each of the peak widths can be increased by lowering the reflectivity of the ends of the tubes.

\section{Level splitting}

To compare the quantum mechanical and acoustic systems, the eigenfrequencies and resonant frequencies need to be expressed in terms of reflectivity. Acoustically, the resonant frequencies are measured for each of the hole sizes. From Eq. (20) the hole size can be related to the reflectivity. As in Eq. (6), we relate the quantum mechanical eigenfrequencies to the wave numbers through the dispersion relation, $f=v k / 2 \pi$. The wave numbers are found as a function of the delta function barrier strength in Eq. (27). The delta function barrier strength is expressed in terms of the reflectivity in Eq. (18).

The graph is shown in Fig. 5 along with the eigenfrequencies found from the perturbative approach. Figure 5 shows that for both the quantum and acoustic systems, level splitting is present. As the reflectivity increases, the odd orders shift to lower frequencies. Once the reflectivity reaches unity, the odd orders merge with the lower even orders. The good agreement between the analytic results and the measurements show that a mapping exists. The perturbative calculation agrees well with the analytic solution at weak perturbations, but deviates at stronger perturbations.

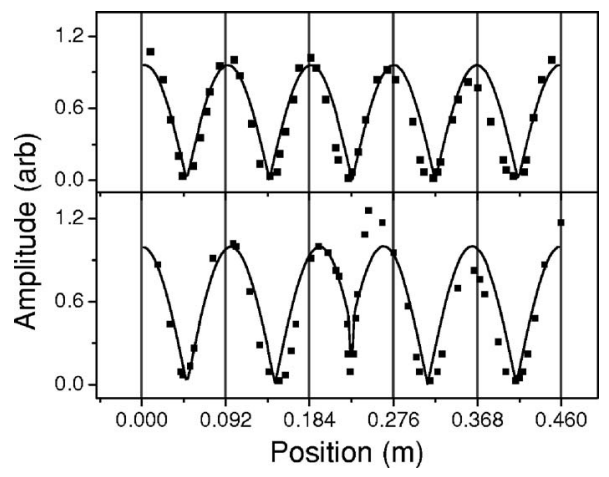

Fig. 6. The absolute value of the pressure wave is measured and compared with the quantum mechanical result as a function of position. Experimental data is given by squares, and the solid line represents the quantum mechanical result (see text). The top graph depicts the fifth harmonic of the long tube with no central disk. The bottom graph is the fifth harmonic when a disk with a $24 \mathrm{~mm}$ hole is introduced to the system.

\section{Wave function and standing wave}

The spatial dependence of the acoustic standing waves can be compared to the quantum mechanical wave functions. The rectified microphone signal is proportional to the absolute value of the acoustic pressure. The gradient of the displacement wave is proportional to the pressure wave. Because the acoustic displacement wave is analogous to the quantum mechanical wave, we compare the measured signal to the absolute value of the gradient of the quantum mechanical wave function (see Fig. 6).

The top graph of Fig. 6 depicts the waveform for the fifth harmonic of the $2 a$ long tube without an interior disk (or, in the quantum case, no delta well.) The bottom graph depicts the same harmonic, but with a $24 \mathrm{~mm}$ holed disk placed in the center of the tube. As in the quantum system, the perturbation causes a kink in the waveform at the perturbation. From our level splitting measurement it is expected that the sound frequency decreases when a disk with a hole is introduced. The observed increase in wavelength (for the bottom graph as compared to the top graph) confirms this behavior.

\section{PEDAGOGICAL NOTES}

Our experiment has been built in stages by undergraduate students in an advanced laboratory course. The motivation of the first stage was to find resonant frequencies in a single tube. Other approaches included resonances in rectangular plexiglass boxes, aluminum tubes, and steel honeycomb structures. Time-dependent sound pulses were also propagated through the tube as an analogy to slow light propagation, ringdown cavities, and time-dependent quantum mechanical problems. The second stage consisted of exploring the systematics of the resonances and automating the data taking procedure. In particular, pouring liquid nitrogen over the tubes shifts the resonances; filling the tube with He gas gave inconclusive results. The automation was done using Basic and LabView. The third stage involved combining multiple cavities, which lead to level splitting. Multiple approaches for reflection were used. Thin membranes without holes were less successful. More cavities were added. For each additional cavity an additional resonance peak forms. For six coupled cavities the resonances merge into groups similar to band structure. Currently, students are exploring 
ways to change the direction the resonances shift, much like the effect of using a delta potential barrier instead of a delta potential well causes the eigenenergies to shift in the opposite direction. The current idea is to replace the smaller diameter holed disks with thin tubes of diameters greater than the tube sections. These tubes will connect the longer tube sections like the holed disks.

All of the previously mentioned system changes were originally posed as a problem for student exploration. The stepwise development and broad range of possible exploration makes this experiment ideally suited for advanced laboratories.

\section{ACKNOWLEDGMENTS}

The authors thank Kees Uiterwaal, Paul Burrow, and Cliff Bettis for fruitful discussions.

\section{APPENDIX: THE REFLECTION COEFFICIENT OF A DELTA POTENTIAL WELL}

A single delta function well is described by

$$
V=-\alpha \delta(x) \text {, }
$$

where $\alpha>0$. The trial solutions for this single delta function well are

$$
\varphi_{\mathrm{I}}(x)=A \exp (i k x)+B \exp (-i k x)
$$

and

$$
\varphi_{\text {II }}(x)=C \exp (i k x),
$$

where region $\mathrm{I}$ is to the left and region II is to the right of the delta function. To find the reflectivity, we assume that no particle can travel toward the delta well from the right, so the term $\exp (-i k x)$ is absent in region II. The boundary conditions at the delta function are

$$
\varphi_{\mathrm{I}}\left(x_{0}\right)=\varphi_{\mathrm{II}}\left(x_{0}\right) \text {, }
$$

$$
\frac{d}{d x} \varphi_{\mathrm{II}}\left(x_{0}\right)-\frac{d}{d x} \varphi_{\mathrm{I}}\left(x_{0}\right)=-\frac{2 m \alpha}{\hbar^{2}} \varphi\left(x_{0}\right) .
$$

Matching these boundary conditions yields

$$
B=-\frac{m \alpha}{i k \hbar^{2}+m \alpha} A,
$$

which gives the reflection coefficient

$$
r \equiv \frac{B}{A}=-\frac{m \alpha}{i k \hbar^{2}+m \alpha} .
$$

${ }^{\text {a) }}$ Author to whom correspondence should be addressed. Electronic mail: shilber1@bigred.unl.edu

${ }^{1}$ Richard L. Liboff, Introductory Quantum Mechanics (Addison-Wesley, San Francisco, CA, 2003).

${ }^{2}$ John S. Townsend, A Modern Approach to Quantum Mechanics (University Science Books, Sausalito, CA, 2000).

${ }^{3}$ David J. Griffiths, Introduction of Quantum Mechanics (Prentice Hall, Upper Saddle River, NJ, 1995).

${ }^{4}$ Claude Cohen-Tannoudji, Bernard Diu, and Franck Laloë, Quantum Mechanics (Hermann, Paris, 1977).

${ }^{5}$ Eugen Merzbacher, Quantum Mechanics (Wiley, New York, NY, 1961).

${ }^{6}$ Lawrence E. Kinsler, Austin R. Frey, Alan B. Coppens, and James V. Sanders, Fundamentals of Acoustics (Wiley, New York, 1982), pp. 98105.

${ }^{7}$ Herbert Goldstein, Classical Mechanics (Addison-Wesley, San Francisco, CA, 1959), pp. 355-359.

${ }^{8}$ Reference 6, pp. 231-234.

${ }^{9}$ Paul R. Berman, Atom Interferometry (Academic Press, San Diego, CA, 1997).

${ }^{10}$ Haym Kruglak and Charles C. Kruse, "A visual method for demonstrating refraction of sound," Am. J. Phys. 8, 260-261 (1940).

${ }^{11}$ J. N. Munday, C. Brad Bennett, and W. M. Robertson, "Band gaps and defect modes in periodically structured waveguides," J. Acoust. Soc. Am. 112, 1353-1358 (2002).

${ }^{12}$ Eugene Hecht, Optics (Addison-Wesley, San Francisco, CA, 2002).

${ }^{13}$ Reference 4, pp. 69-70.

${ }^{14}$ Reference 4, pp. 1100-1102.

${ }^{15}$ Yoav Peleg, Reuven Pnini, and Elyahi Zaaror, Schaum's Outline of Theory and Problems of Quantum Mechanics (McGraw-Hill, New York, 1998), pp. 181-182. 